\title{
Explicit solution of the large amplitude transverse vi- brations of a flexible string under constant tension
}

\begin{abstract}
This paper presents the analytical simulation of string with large amplitudes using the Variational Iteration Method (VIM) and Hamiltonian Approach (HA). In order to verify the precision of the presented methods, current results were compared with He's Variational Approach and Runge-Kutta $4^{\text {th }}$ order. It has been found that these methods are well suited for a range of parameters and the approximate frequencies and periodic solutions show a good agreement with other techniques. The results show that both methods can be easily extended to other nonlinear oscillations and it can be predicted that both methods can be found widely applicable in engineering.
\end{abstract}

\section{Keywords}

Variational Iteration Method; Hamiltonian Approach; Nonlinear oscillation; Non-linear vibration string

\author{
Reza Taghipour ${ }^{a}$, \\ Tohid Akhlaghi', \\ Ali Nikkar ${ }^{b}{ }^{*}$ \\ ${ }^{\mathrm{a}}$ Department of Civil Engineering, University \\ of Mazandaran, Babolsar, Iran \\ ${ }^{b}$ Faculty of Civil Engineering, \\ University of Tabriz, Tabriz, Iran \\ Received in 14 May 2013 \\ In revised form 30 June 2013 \\ *Author email: ali.nikkar@yahoo.com
}

\section{INTRODUCTION}

Study of nonlinear problems in strings with large amplitude is a very important research area in many fields of physics and engineering. Long cables and wires are extensively used in many mechanical systems and structures such as cranes, ships, offshore platforms and bridges. The study of nonlinear vibration of strings (cables and wires) with large amplitude is in practice very complicated. Primarily, the force data like air flow speed, direction and turbulence characteristic is, as a rule, difficult to definitely specify. Further, the mechanical characteristic of cables like bending stiffness and damping effects depend on a number of parameters (Omran et al., 2013).

On the other hand, with the rapid development of nonlinear science, there appears an everincreasing interest of scientists in the analytical asymptotic techniques for nonlinear problems, and several analytical approximation methods have been developed. such as: variational iteration method (He, 2007a; Barari et al., 2011, Saadati et al., 2009), homotopy perturbation method (Torabi and Yaghoobi, 2011; Torabi et al., 2011; Saravi et al., 2013), homotopy analysis method (Liao, 2003; Khan et al., 2012)and some other methods (Bagheri et al. 2014; Nikkar et al., 2012,2014; Ghasempoor et al., 2012; Akbarzade and Khan, 2012; Alinia et al., 2011; Ganji, 2012; Torabi et al., 
2012; Sheikholeslami et al., 2012a; 2012b; Bayat et al., 2011;2012; Rafieipour et al., 2012; Marinca and Herisanu, 2010; Salehi et al., 2012; Hamidi et al., 2012)

The main objective of the present study is to propose two analytical methods, namely the Variational Iteration Method (VIM) and Hamiltonian Approach (HA) which were introduced by He (He, $1999 ; 2010)$ to solve the nonlinear differential equation of the large deformation of string with large amplitudes. The Effectiveness and convenience of the methods is revealed in comparisons with the other solution techniques.

The results reveal that these methods are very effective and convenient in predicting the solution of such problems, and it is predicted that the VIM and Hamiltonian Approach (HA) can find a wide application in new engineering problems.

\section{THE GOVERNING EQUATION OF STRINGS WITH LARGE AMPLITUDE}

Considering the differential equation of large amplitude transverse vibrations of a flexible string under constant tension as shown in Fig. 1.

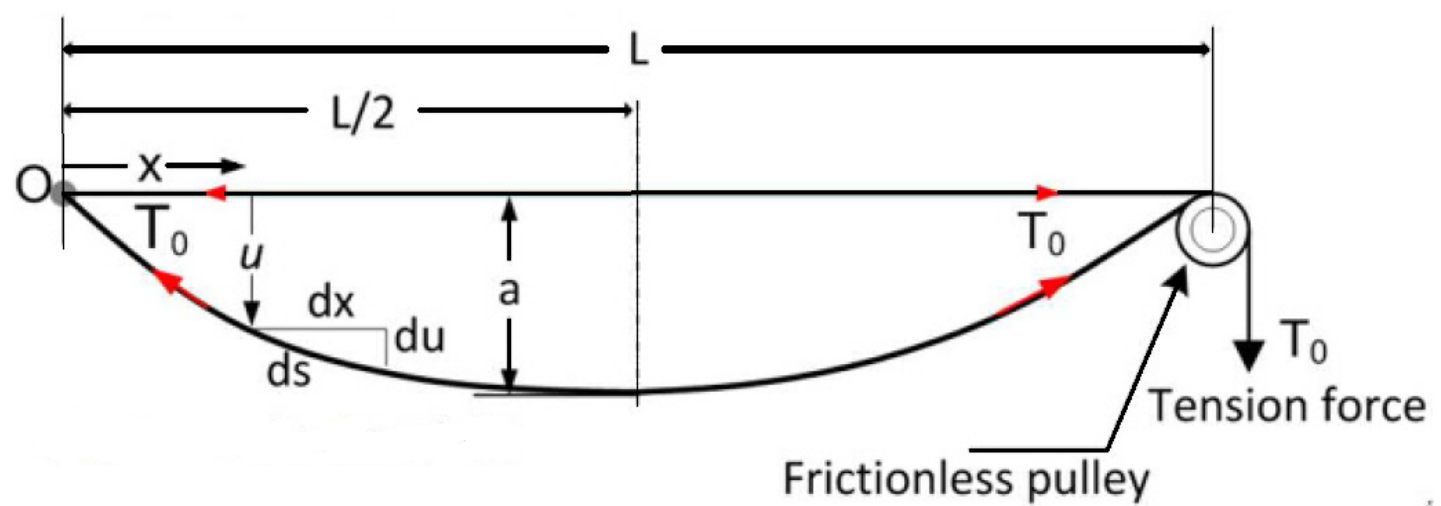

Figure 1 Schematic of a constant tension vibrating string with large amplitude (Omran et al., 2013).

The governing differential equation of large amplitude vibrations of a constant tension string of this form is written as:

$$
c^{2} \frac{\partial u^{2}}{\partial x^{2}}=\left(1+\frac{\partial u^{2}}{\partial x^{2}}\right)^{2} \frac{\partial u^{2}}{\partial t^{2}}
$$

where $\mathrm{u}(\mathrm{x}, \mathrm{t})$ is the transverse displacement amplitude relative to the spatial $\mathrm{x}$ and temporal $\mathrm{t}$ coordinates, $\mathrm{c}=\tau_{0} / \rho_{0}$ is the velocity of transverse wave with $\tau_{0}$ and $\rho_{0}$ being the tension and the mass per unit length, respectively. Let's also consider that the transverse displacement is expressed as:

$$
u(x, t)=V(t) \sin \left(\frac{\pi}{L} x\right)
$$


Substituting Eq. (2) into Eq. (1) and averaging over the string length $L$ (i.e. a Galerkin procedure is performed to take the multiplication of Eq. (2) by Eq. (1)) and integrating such equation over $x$ from 0 to $L$ results in an ordinary second order differential equation as:

$$
\frac{d^{2} \varphi}{d t^{2}}+\frac{\alpha \varphi}{1+\frac{\varphi^{2}}{4}+\frac{\varphi^{4}}{8}}=0
$$

With initial conditions:

$$
\varphi(0)=A, \quad \dot{\varphi}(0)=0
$$

where $\varphi$ and $t$ are generalized dimensionless displacement and time variables. Also we have:

$$
\varphi(t)=\frac{\pi}{L} V(t), \quad \alpha=\left(\frac{\pi c}{L}\right)^{2}
$$

\section{DESCRIPTION OF THE VARIATIONAL ITERATION METHOD}

To clarify the idea of the proposed method for solution of the large deformation of string with large amplitudes, the basic concept of Variational Iteration Method is firstly treated. We consider the following general differential equation,

$$
L u+N u=g(t)
$$

Where, $\mathrm{L}$ is a linear operator, and $\mathrm{N}$ a nonlinear operator, $\mathrm{g}(\mathrm{t})$ an inhomogeneous or forcing term. According to the variational iteration method, we can construct a correct functional as follows:

$$
u_{n+1}(t)=u_{n}(t)+\int_{0}^{t} \lambda\left(L u_{n}(\tau)+N \tilde{u}_{n}(\tau)-g(\tau)\right) d \tau
$$

Where $\lambda$ is a general Lagrange multiplier, which can be identified optimally via the variational theory, the subscript $n$ denotes the nth approximation, $\widetilde{u}_{n}$ is considered as a restricted variation, i.e. $\delta \tilde{u}_{n}=0$.

For linear problems, its exact solution can be obtained by only one iteration step due to the fact that the Lagrange multiplier can be exactly identified. In this method, the problems are initially approximated with possible unknowns and it can be applied in non-linear problems without linearization or small parameters. The approximate solutions obtained by the proposed method rapidly converge to the exact solution. 


\section{IMPLEMENTATION OF VARIATIONAL ITERATION METHOD}

Now, we can solve the Eq. (3). Assume that the angular frequency of the system (3) is $\omega$, we have the following linearized equation:

$$
\ddot{\varphi}+\omega^{2} \varphi=0
$$

So we can rewrite Eq. (3) in the form:

$$
\ddot{\varphi}+\omega^{2} \varphi+f(\varphi)=0
$$

Where

$$
f(\varphi)=-\omega^{2} \varphi+\frac{\alpha \varphi}{1+\frac{\varphi^{2}}{4}+\frac{\varphi^{4}}{8}}=0
$$

Applying the proposed method, the following iterative formula is formed as:

$$
\varphi_{n+1}(t)=\varphi_{n}(t)+\int_{0}^{t} \lambda\left(\ddot{\varphi}_{n}(\tau)+\omega^{2} \varphi_{n}(\tau)+f(\varphi(\tau)) d \tau\right.
$$

Its stationary conditions can be obtained as follows:

$$
\begin{aligned}
& 1-\left.\lambda^{\prime}\right|_{\tau=t}=0, \\
& \left.\lambda\right|_{\tau=t}=0, \\
& \lambda^{\prime \prime}+\left.\omega^{2} \lambda\right|_{\tau=t}=0 .
\end{aligned}
$$

The Lagrange multiplier, therefore, can be identified as;

$$
\lambda=\frac{1}{\omega} \sin \omega(\tau-t)
$$

By substituting this identified multiplier into Eq. (11), we come to:

$$
\varphi_{n+1}(t)=\varphi_{n}(\tau)+\frac{1}{\omega} \int_{0}^{t} \sin \omega(\tau-t)\left(\frac{d^{2} \varphi_{n}(\tau)}{d t^{2}}+\frac{\alpha \varphi_{n}(\tau)}{1+\frac{\varphi_{n}(\tau)^{2}}{4}+\frac{\varphi_{n}(\tau)^{4}}{8}}\right) d \tau
$$

Assuming its initial approximate solution has the form:

Latin American Journal of Solids and Structures 11 (2014) 545 - 555 


$$
\varphi_{0}(t)=A \cos (\omega t)
$$

And substituting Eq. (15) into Eq. (3) leads to the following residual:

$$
R_{0}(t)=-A \omega^{2} \cos (\omega t)+\frac{\alpha A \cos (\omega t)}{1+\frac{A^{2} \cos (\omega t)^{2}}{4}+\frac{A^{4} \cos (\omega t)^{4}}{8}}
$$

By the formulation (14), we can obtain

$$
\varphi_{1}(t)=\varphi_{0}(t)+\frac{1}{\omega} \int_{0}^{t} \sin \omega(\tau-t)\left(R_{0}(\tau)\right) d \tau
$$

In the same manner, the rest of the components of the iteration formula can be obtained. In order to ensure that no secular terms appear in $u_{1}$, resonance must be avoided. To do so, the coefficient of $\cos (\omega t)$ in Eq. (16) requires being zero, i.e.,

$$
\omega_{V I M}=\frac{2}{A} \sqrt{2 \alpha \arctan \left(A+\frac{1}{2} A^{2}\right)-2 \alpha \arctan \left(A+\frac{1}{4} A^{4}\right)}
$$

\section{BASIC CONCEPT OF HAMILTONIAN APPROACH}

Previously, He (He, 2002) had introduced the energy balance method based on collocation and Hamiltonian. Recently, in 2010 it was developed into the Hamiltonian approach (He, 2010). This approach is a kind of energy method with a vast application in conservative oscillatory systems. In order to clarify this approach, consider the following general oscillator:

$$
\ddot{u}+f(u, \dot{u}, \ddot{u})=0
$$

With initial conditions:

$$
u(0)=A, \quad \dot{u}(0)=0
$$

Oscillatory systems contain two important physical parameters, i.e. the frequency $\omega$ and the amplitude of oscillation A. It is easy to establish a variational principle for Eq. (19), which reads;

$$
J(u)=\int_{0}^{\frac{T}{4}}\left(-\frac{1}{2} \dot{u}^{2}+F(u)\right) d t
$$

Where $\mathrm{T}$ is period of the nonlinear oscillator and $\frac{\partial F}{\partial u}=f$ 
In the Eq. (21), $\dot{u}^{2} / 2$ is kinetic energy and $F(u)$ potential energy, so the Eq. (21) is the least Lagrangian action, from which we can immediately obtain its Hamiltonian, which reads:

$$
H(u)=-\frac{1}{2} \dot{u}^{2}+F(u)=\text { CONSTANT }
$$

From Eq. (22), we have;

$$
\frac{\partial H}{\partial A}=0
$$

Introducing a new function, $\bar{H}(u)$, defined as:

$$
\bar{H}(\varphi)=\int_{0}^{\frac{T}{4}}\left(-\frac{1}{2} \dot{u}^{2}+F(u)\right) d t=\frac{1}{4} T H
$$

Eq. (23) is, then, equivalent to the following one;

$$
\frac{\partial}{\partial A}\left(\frac{\partial \bar{H}}{\partial T}\right)=0
$$

Or

$$
\frac{\partial}{\partial A}\left(\frac{\partial \bar{H}}{\partial\left(\frac{1}{\omega}\right)}\right)=0
$$

From Eq. (26) we can obtain approximate frequency-amplitude relationship of a nonlinear oscillator.

\section{THE APPLICATION OF HAMILTONIAN APPROACH}

To illustrate the basic procedure of the present method, the Hamiltonian of Eq. (5) can be written in the form:

$$
H(\varphi)=-\frac{1}{2} \dot{\varphi}^{2}+2 \alpha \arctan \left(1+\frac{1}{2} \varphi^{2}\right)
$$

Introducing a new function, $\bar{H}(\varphi)$. Integrating Eq. (27) with respect to t from 0 to $\mathrm{T} / 4$, we obtain:

$$
\bar{H}(\varphi)=\int_{0}^{\frac{T}{4}}\left(-\frac{1}{2} \dot{\varphi}^{2}+2 \alpha \arctan \left(1+\frac{1}{2} \varphi^{2}\right)\right) d t
$$


Assuming that the solution can be expressed as $\varphi=A \cos (\omega t)$ and substituting it into Eq. (28) yields:

$$
\bar{H}(\varphi)=\int_{0}^{\frac{T}{4}}\left(-\frac{1}{2} A^{2} \omega^{2} \sin (\omega t)^{2}+2 \alpha \arctan \left(1+\frac{1}{2} A^{2} \cos (\omega t)^{2}\right)\right) d t
$$

Setting

$$
\frac{\partial}{\partial A}\left(\frac{\partial \bar{H}}{\partial\left(\frac{1}{\omega}\right)}\right)=0
$$

So the frequency can be approximated as:

$$
\omega_{H A}=\frac{2}{A} \sqrt{2 \alpha \arctan \left(A+\frac{1}{2} A^{2}\right)-2 \alpha \arctan \left(A+\frac{1}{4} A^{4}\right)}
$$

Note: One can expand $\arctan \left(A+\frac{1}{2} A^{2}\right)$ into Taylor series for small A, and compare the result with that obtained by perturbation method.

\section{RESULTS AND DISSCUSIONS}

A mathematical model describing the process of large amplitude transverse vibrations of a flexible string under constant tension is proposed. Two efficient analytical methods are applied to solve the dynamic model of the large amplitude non-linear oscillation equation. In order to verify the precision of the methods, current results were compared with Runge-Kutta $4^{\text {th }}$ order method in Table 1 and 2. It is observable that our results are in excellent agreement with the results provided by Runge-Kutta $4^{\text {th }}$ order. The behavior of $\varphi(A, t)$ obtained by VIM and HA at $a=2$ is shown in Figs. 2 and 3. To further illustrate the accuracy of presented methods, the plot of the results are presented and compared in Figs. 4-6. All plots are done for a constant value of $\alpha=1$ and varying amplitude

\begin{tabular}{|c|c|c|c|c|c|}
\hline A & VIM & HA & RKF & ERROR(VIM) & $\operatorname{ERROR}(\mathrm{HA})$ \\
\hline 1 & 0.8899905311 & 0.914508356 & 0.90951228723 & 0.01952175613 & -0.00499606877 \\
\hline 2 & 1.8878586871 & 2.040047936 & 1.93700826192 & 0.04914957482 & -0.10303967408 \\
\hline 3 & 2.7467435860 & 3.029905311 & 2.97185868708 & 0.22511510108 & -0.05804662392 \\
\hline 4 & 3.8690203289 & 4.017121304 & 3.98646608228 & 0.11744575338 & -0.03065522172 \\
\hline 5 & 4.9267435859 & 5.010179315 & 4.99267435859 & 0.06593077269 & -0.01750495641 \\
\hline 10 & 9.9690826192 & 10.00162672 & 9.99902032887 & 0.02993770967 & -0.00260639113 \\
\hline
\end{tabular}
values $\mathrm{A}=0.2,1$ and 5 .

Table 1 Comparison of response of the system $\varphi(A, t)$ obtained by the presented methods with numerical results when $t=0.5(s), \alpha=1$ 
Table 2 Comparison of response of the system $\varphi(A, t)$ obtained by the presented methods with numerical results when $t=1(s), \alpha=2$

\begin{tabular}{|c|c|c|c|c|c|}
\hline$A$ & $V I M$ & $H A$ & $R K F$ & $\operatorname{ERROR}(V I M)$ & $\operatorname{ERROR}(H A)$ \\
\hline 1 & 0.265794960 & 0.382722526 & 0.31822973907 & 0.05243477906 & -0.06449278693 \\
\hline 2 & 2.116832133 & 2.327908002 & 2.23752825750 & 0.12069612450 & -0.09037974449 \\
\hline 3 & 2.612042483 & 3.242032233 & 2.96872183773 & 0.35667935473 & -0.27331039527 \\
\hline 4 & 3.427675192 & 4.137655192 & 3.89055794960 & 0.46288275760 & -0.24709724240 \\
\hline 5 & 4.701229739 & 5.081628040 & 4.94111051404 & 0.23988077504 & -0.14051752596 \\
\hline 10 & 9.510341463 & 10.01301620 & 9.99216000549 & 0.48181854249 & -0.02085619451 \\
\hline
\end{tabular}

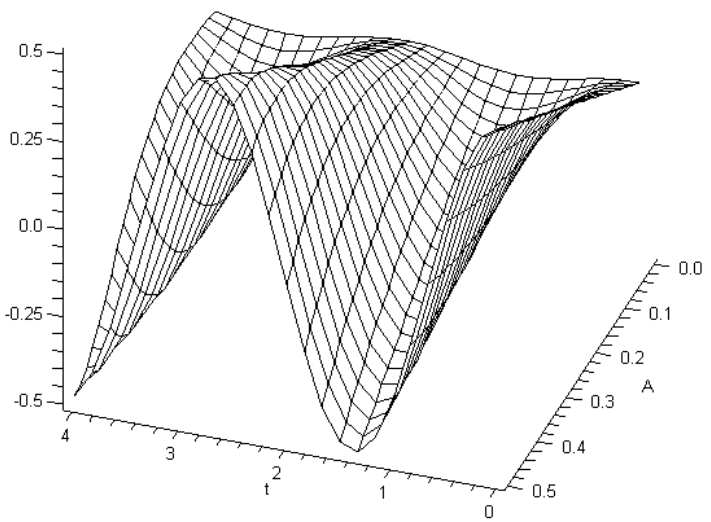

Figure 2 VIM deflection at $\alpha=2$

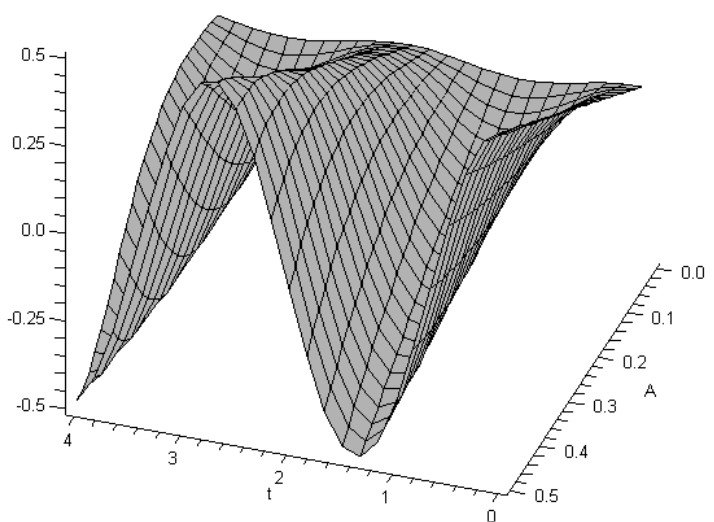

Figure 3 HA deflection at $\alpha=2$

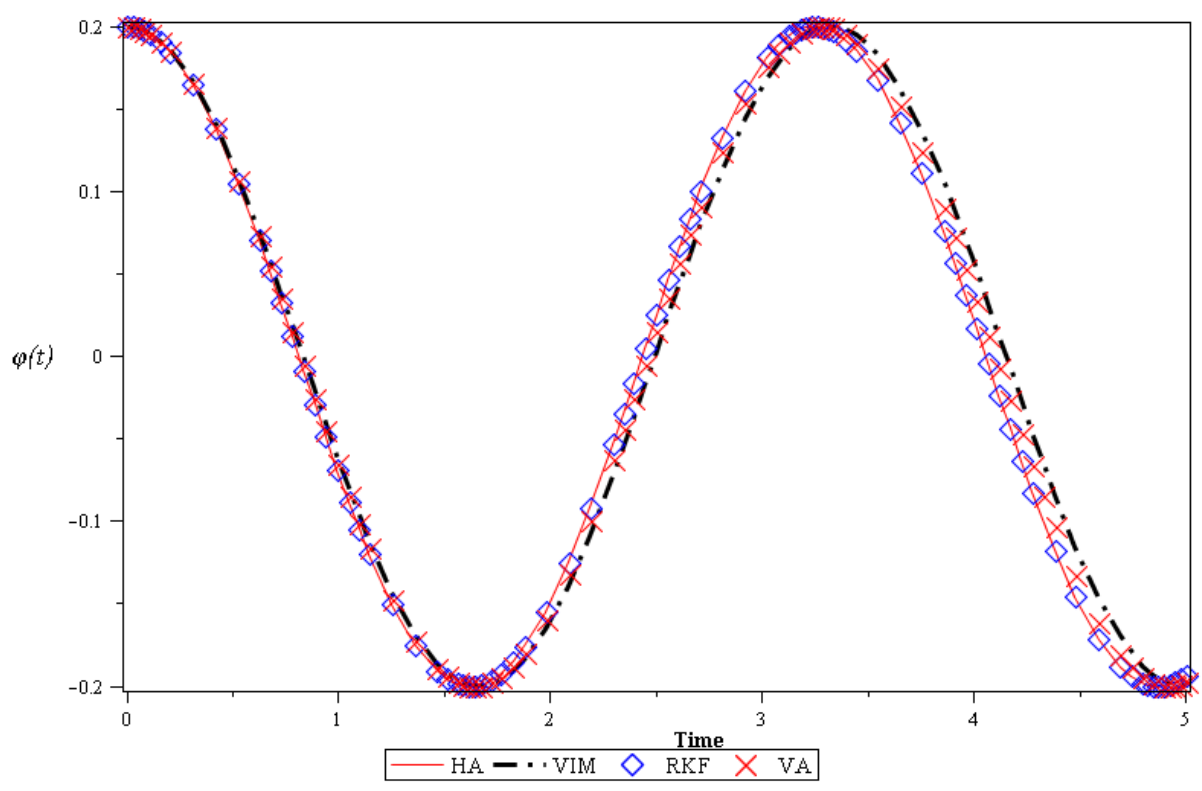

Figure 4 Comparison between Variational Iteration (VIM), Hamiltonian Approach (HA) and Variational Approach (VA) periodic solutions versus time with Runge-Kutta $4^{\text {th }}$ order for $\alpha=1$ and $A=0.2$. 


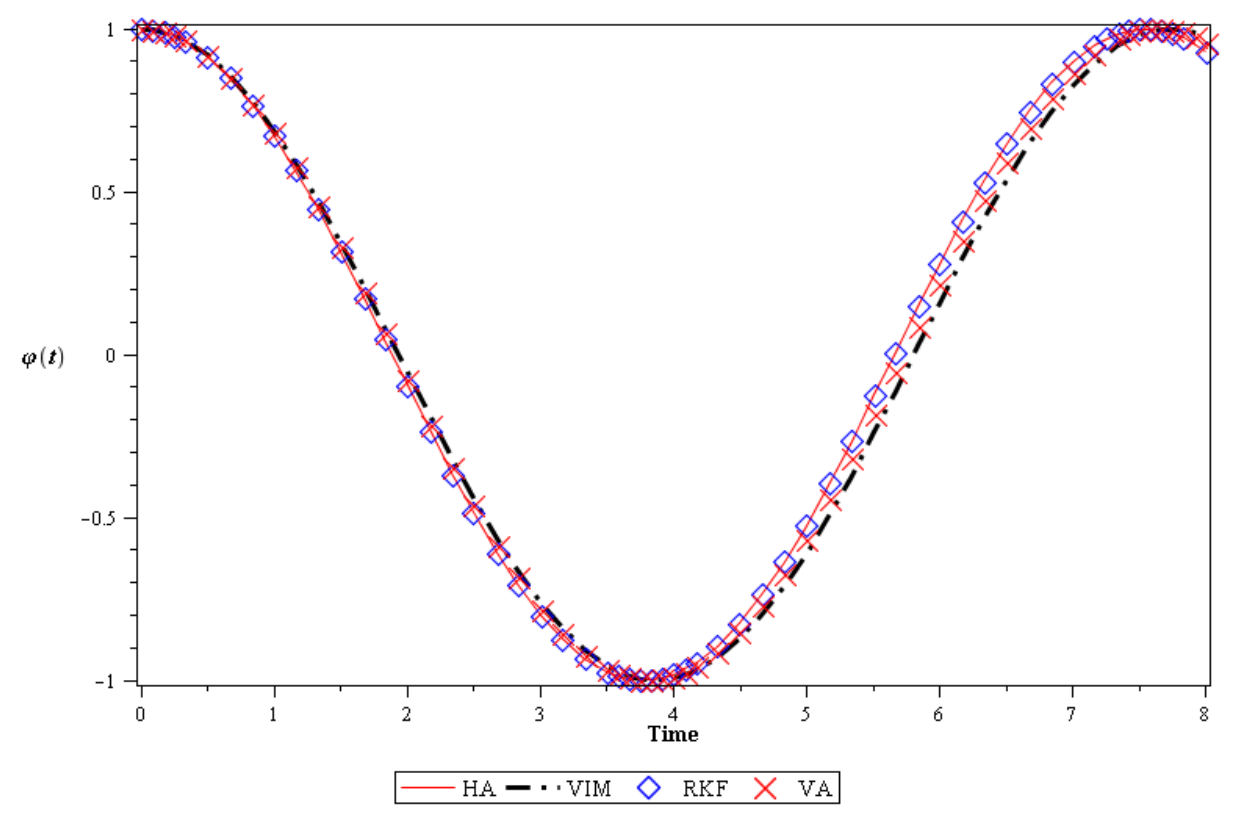

Figure 5 Comparison between Variational Iteration (VIM), Hamiltonian Approach (HA) and Variational Approach (VA) periodic solutions versus time with Runge-Kutta $4^{\text {th }}$ order for $\alpha=1$ and $A=1$.

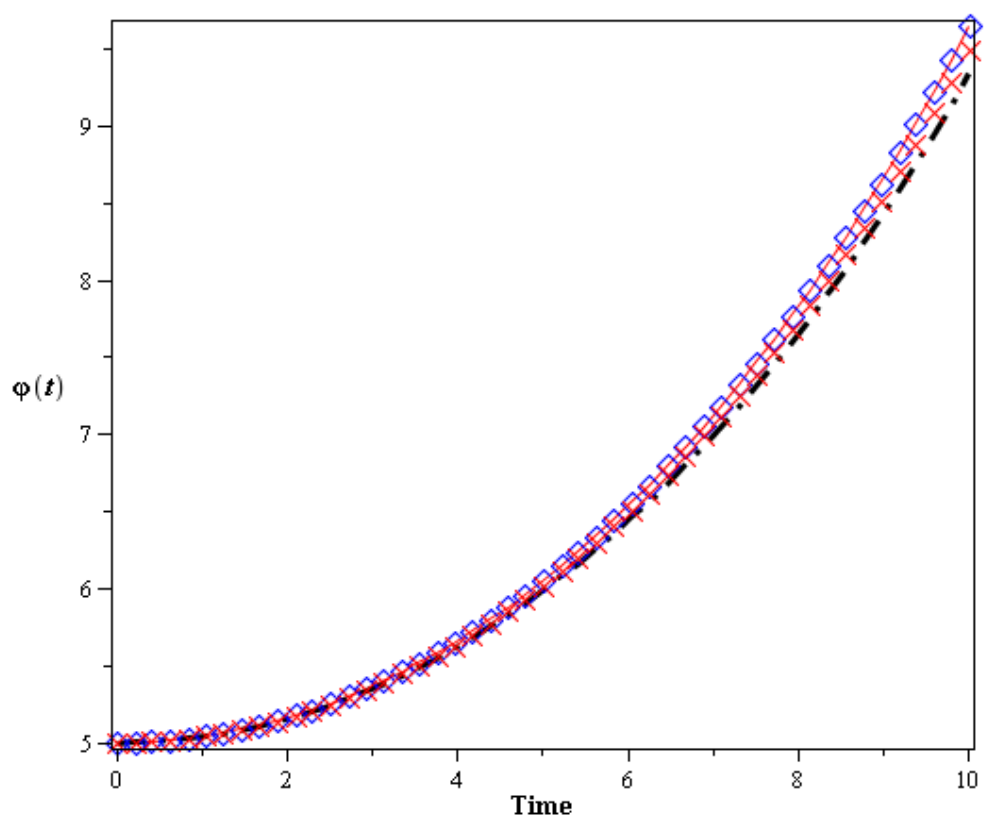

Figure 6 Comparison between Variational Iteration (VIM), Hamiltonian Approach (HA) and Variational Approach (VA) periodic solutions versus time with Runge-Kutta $4^{\text {th }}$ order for $\alpha=1$ and $A=5$. 


\section{CONCLUSIONS}

In this work, we proposed a mathematical model describing the process of large amplitude transverse vibrations of a flexible string under constant tension. Hamiltonian approach and efficient approximate method (VIM) is employed to derive the nonlinear vibration of constant-tension string. The frequency of both methods is exactly the same. Comparing with numerical Results, it is shown that the approximate analytical solutions are in very good agreement with the corresponding solutions and presented methods are powerful mathematical tools, very effective, convenient and adequately accurate for study of nonlinear oscillators in physics and engineering problems.

\section{References}

Akbarzade, M., Khan, Y., (2012). Dynamic model of large amplitude non-linear oscillations arising in the structural engineering: Analytical solutions. Mathematical and Computer Modelling, 55:480-489.

Alinia, M., Ganji, D. D., Gorji-Bandpy, M., (2011). Numerical study of mixed convection in an inclined two sided lid driven cavity filled with nanofluid using two-phase mixture model. International Communications in Heat and Mass Transfer, 38(10): 1428-1435.

Barari, A., Kaliji, H. D., Ghadimi, M., Domairry, G., (2011). Non-linear vibration of Euler-Bernoulli beams. Latin American Journal of Solids and Structures 8(2):139-148.

Bagheri, S., Nikkar, A., Ghaffarzadeh, H., (2014). Study of nonlinear vibration of Euler-Bernoulli beams by using analytical approximate techniques. Latin American Journal of Solids and Structures 11(1): 157-168.

Bayat, Mahmoud., Pakar, Iman., Bayat, Mahdi., (2011). Analytical study on the vibration frequencies of tapered beams. Latin American Journal of Solids and Structures 8(2):149-162.

Bayat, M., Pakar, I., Domairry, G., (2012). Recent developments of some asymptotic methods and their applications for nonlinear vibration equations in engineering problems: a review. Latin American Journal of Solids and Structures, 9 (2): 1-93.

Ganji, D. D. (2012). A semi-Analytical technique for non-linear settling particle equation of Motion. Journal of Hydro-environment Research 6(4): 323-327.

Ghasempour, S., Vahidi, J., Nikkar A., Mighani, M., (2012). Analytical Approach to Some Highly Nonlinear Equations by Means of the RVIM. Research Journal of Applied Sciences, Engineering and Technology, 5(1): 339-345.

Hamidi, S.M., Rostamiyan, Y., Ganji, D.D., Fereidoon, A., (2012). A novel and developed approximation for motion of a spherical solid particle in plane coquette fluid flow. Advanced Powder Technology, http://dx.doi.org/10.1016/j.apt.2012.07.00

He, J.H., (1999). Variational iteration method - a kind of non-linear analytical technique: Some examples. Int. J. Nonl. Mech., 34:699-708.

He, J. H., (2002). Preliminary report on the energy balance for nonlinear oscillations. Mechanics Research Communications, 29:107-111.

He, J. H. (2006). Some asymptotic methods for strongly nonlinear equations. International Journal of modern Physics B, 20(10):1141-1199.

He, J.H., (2007a). Variational iteration method - some recent results and new interpretations. J Comput Appl Math, 20(1):3-17.

He, J. H. (2007b). Variational approach for nonlinear oscillators. Chaos, Solitons and Fractals, 34:1430-1439.

He, J. H., (2010). Hamiltonian approach to nonlinear oscillators. Physics Letters A, 374: 2312-2314. 
He, J.H., (2012). Asymptotic methods for solitary solutions and compactions. Abstract and applied analysis, doi:10.1155/2012/916793

Khan, Y., Taghipour, R., Fallahian, M., Nikkar, A., (2012). A new approach to modified regularized long wave equation. Neural Computing and Applications, doi:10.1007/s00521-012-1077-0

Liao, S. J., (2003). Beyond perturbation: introduction to homotopy analysis method. Chapman \& Hall/CRC Press, Boca Raton.

Marinca, V., Herisanu, N., (2010) Optimal homotopy perturbation method for strongly nonlinear differential equations. Nonlinear Science Letters A, 1(3): 273-280.

Nikkar, A., Mighani, Z., Saghebian, S.M., Nojabaei, S.B., Daie, M., (2012). Development and Validation of an Analytical Method to the Solution of Modelling the Pollution of a System of Lakes. Research Journal of Applied Sciences, Engineering and Technology, 5(1):296-302.

Nikkar, A., Bagheri, S., Saravi, M., (2014). Study of nonlinear vibration of Euler-Bernoulli beams by using analytical approximate techniques. Latin American Journal of Solids and Structures 11(2): 320-329.

Omran, M. P., Amani, A., Lemu, H. G., (2013). Analytical approximation of nonlinear vibration of string with large amplitudes. Journal of Mechanical Science and Technology, 27(4): 981-986.

Rafieipour, H., Lotfavar, A., Mansoori, M.H., (2012). New Analytical Approach to Nonlinear Behavior Study of Asymmetrically LCBs on Nonlinear Elastic Foundation under Steady Axial and Thermal Loading. Latin American Journal of Solids and Structures, 9(5):531-545.

Saadati, R., Dehghan, M., Vaezpour, S. M., Saravi, M., (2009). The convergence of He's variational iteration method for solving integral equations. Computers \& Mathematics with Applications, 58 (11): 2167-2171.

Salehi, P., Yaghoobi, H., Torabi, M., (2012). Application of the Differential Transformation Method and Variational Iteration Method to Large Deformation of Cantilever Beams under Point Load. Journal of Mechanical Science and Technology, 26 (9):2879-2887.

Saravi, M., Hermaan, M., Ebarahimi khah, H., (2013). The comparison of homotopy perturbation method with finite difference method for determination of maximum beam deflection. Journal of Theoretical and Applied Physics, 7:8, doi:10.1186/2251-7235-7-8

Sheikholeslami, M., Soleimani, S., Gorji-Bandpy, M., Ganji, D. D., Seyyedi, S. M., (2012a). Natural convection of nanofluids in an enclosure between a circular and a sinusoidal cylinder in the presence of magnetic field. International Communications in Heat and Mass Transfer, 39(9): 1435-1443.

Sheikholeslami, M., Gorji-Bandpy, M., Ganji, D. D., (2012b). Magnetic field effects on natural convection around a horizontal circular cylinder inside a square enclosure filled with nanofluid. International Communications in Heat and Mass Transfer, 39(7): 978-986

Torabi, M., Yaghoobi, H., (2011). Novel Solution for Acceleration Motion of a Vertically Falling Spherical Particle by HPM-Pade Approximant. Advanced Powder Technology, 22:674-677.

Torabi, M., Yaghoobi, H., Saedodin, S., (2011). Assessment of Homotopy Perturbation Method in Nonlinear Convective-Radiative Non-Fourier Conduction Heat Transfer Equation with Variable Coefficient. Thermal Science, 15:263-274.

Torabi, M., Yaghoobi, H., Aziz, A., (2012). Analytical Solution for Convective-Radiative Continuously Moving Fin with Temperature Dependent Thermal Conductivity. International Journal of Thermophysics, 33:924-941. 